\title{
Competing Polymerization of Actin Skeleton explains Relation between Network Polarity and Cell Movements
}

\author{
B. Nandy and A. Baumgaertner \\ Institut für Festkörperforschung, Forschungszentrum Jülich, 52425 Jülich, Germany
}

(Dated: October 30, 2018)

\begin{abstract}
Based on experimental observations it is known that various biological cells exhibit a persistent random walk during migration on flat substrates. The persistent random walk is characterized by 'stop-and-go' movements : unidirectional motions over distances of the order of several cell diameter are separated by localized short time erratic movements. Using computer simulations the reasons for this phenomena had been unveiled and shown to be attributed to two antagonistic nucleation processes during the polymerization of the cell's actin cytoskeleton : the (ordinary) spontaneous nucleation and the dendritic nucleation processes. Whereas spontaneous nucleations generate actin filaments growing in different directions and hence create motions in random directions, dendritic nucleations provide a unidirectional growth. Since dendritic growth exhibits stochastic fluctuations, spontaneous nucleation may eventually compete or even dominate, which results in a reorientation of filament growth and hence a new direction of cell motion. The event of reorientation takes place at instants of vanishing polarity of the actin skeleton.
\end{abstract}




\section{INTRODUCTION}

Most animal cell types are 'motile' and possess the capacity to move over or through a substrate, and cell migration is a normal occurrence in both normal physiological cases as in the case of diseases. Unlike the phenomena related to 'mobility', where the collision among molecules determine their random movements, the random motion of motile cells requires an energy-consuming mechanism. It is now widely accepted that the basic engine for gliding or crawling locomotion of many living cells is the ATP-supported polymerization of the actin cytoskeleton. Rapidly moving cells can often move over the substrate without microtubules (keratocytes, neutrophils), but crawling motility always requires actin. One of the crucial factors for cell movement is actin polarity [1], which correlates with a persistent random walk during migration. The persistent random walk is characterized by 'stop-and-go' movements : unidirectional motions over distances of the order of several cell diameters are separated by localized short time erratic movements. The main focus of the present study is the cause behind the persistent random walk of a cell, and hence the cause behind the correlated spontaneous change of polarity. It has been seen that cell motility and chemotactic migration are related to specific rearrangements of the actin cytoskeleton. Actin polymerizes into new filaments in regions of the cell that are specified by activated signalling at the plasma membrane [2, 3, 4, 5]. These signals give rise to cell polarity and directional motility. In the absence of chemotactic stimuli, cells exhibit a persistent random walk.

In this work we present results of our investigations on the relation between cytoskeletal actin polymerization and the persistence random walk of a cell. The results are based on the analysis of Monte Carlo simulations of a simple model cell.

\section{MODEL AND SIMULATION TECHNIQUE}

Due to the limitation of information and computational complexity it is not possible to construct the model cell and it's motility including all regulatory proteins with their exact concentration for all types of cells. So we focus on one of the simplest cell which is the keratocyte residing in the epidermis, the most outer layer of the skin. The keratocyte is responsible for the formation of tissue and wound healing, both requiring the cell's ability to autonomously migrate within skin tissue. It is in fact one of the fastest moving cells [6] with a speed of $0.5 \mu \mathrm{m} / \mathrm{s}$. The advantage of choosing keratocyte is that

the crescent-like shape of the cell is almost constant as it moves [7, 8]. The motility of the keratocyte is assumed to be based largely on a continuously remodeling actin network. In our model we neglect 
the cell body, i.e., the nucleus and other organelles. This cell type is known as a cytoplast [9].

\section{A. The description of Membrane and Actin Models}

Membrane. The plasma membrane of a biological cell is a highly complex surface consisting of a lipid bilayer. The complexity of the cell membrane cannot be captured in our simple model membrane. Therefore, similar to the previous successful studies [10, 11, 12], the cell membrane is designed as an elastic two-dimensional ring. Our model membrane is a flexible closed ring embedded on the square lattice. The ring is non-self-avoiding and exibit the usual random walk characteristics. Conformational changes of the ring are achieved by Monte Carlo methods, where a randomly chosen pair of two successive segments of the chain perform a kink jump or hair pin jump to one of the neighbouring sites. Globular Actin (G-actin). The model membrane encloses a fixed number, $N$, of actin molecules. Each actin molecule has a size [6] of about $5 \mathrm{~nm} \times 5 \mathrm{~nm}$ and is located at any of the vertices of the square lattice. The G-actin molecules diffuse freely from one lattice point to another. No excluded volume condition is imposed among G-actin molecules. But it is imposed between membrane and G-actins. So the membrane is impermeable for all actin molecules.

Filament Actin (F-actin). The G-actin monomers form a rigid filament by associating with each other. Within a filament the actin monomers are called F-actin. According to experimental results [13, 14], the filaments are assumed to be chemically coupled to the underlying substrate via membrane proteins. This attachment to the substrate provides the necessary traction forces for the advancement of the cell. Hence, in our model the actin filaments are assumed to be immobile. Excluded volume effects between filaments and diffusing G-actin molecule are neglected, whereas excluded volume effects among filaments and between filaments and membrane are included.

Actin-associated Proteins. A group of actin associated proteins help the remodelling of the actin network, and hence control membrane protrusion. Proteins of the WASP/Scar families activate Arp2/3 protein complexes. These complexes nucleate new actin filaments at the sides of existing filaments. The nascent filaments in the network arise in the form of branches from preexisting filaments. The newly formed filaments elongate at their ends, pushing the membrane at the leading edge forward until they are capped by specific proteins. For example, ADF/Cofilin creates free ends by severing preexisting filaments and promoting depolymerization of free filaments at their opposite ends, and CapZ and gelsolin cap the fast growing ends.

Based on experimental observations it is known that Arp2/3 is activated only close to the membrane. 
Therefore Arp2/3-induced branching processes must be expected to happen only near the membrane. The Arp2/3 molecule is not explicitly taken into account in our model because of its large physiological concentration, in particular near the cell membrane where it becomes activated. In our model, the width of the range of activation is taken to be 10 lattice sites. Accordingly, in our model we perfom branching from an existing filament only if the filament extends to the range of activation near the membrane.

A typical snapshot of the simulated cell is depicted in Fig.1.

\section{B. Probabilities and Reaction Rates}

Polymerization. During the polymerization step the association of G-actin molecules to an existing Factin filament occurs at certain rates at both ends. We adopt the same reaction rates for polymerization and depolymerization as used in a previous work [12], and are listed in Table I. Due to the structural rotational symmetry of a single G-actin molecule, both ends of the F-actin filament are distinguishable : the 'barbed' (or synonymously, 'plus' or 'fast growing') end, and the 'pointed' (or synonymously, 'minus' or 'slow growing') end. The association rates of ATP-bound G-actin to both ends of a filament are different [2, 6, 15, 16], and we denote the rate constants $k_{b}^{+}$and $k_{p}^{+}$for 'barbed' and 'pointed' ends, respectively. The same holds for the depolymerization rates $k_{b}^{-}$and $k_{p}^{-}$, albeit their difference is smaller. Since both ends of a filament are distinguishable, each F-actin filament possesses an intrinsic polarity.

Nucleation. We consider two types of nucleation for F-actin. When nucleation is formed by two G-actin molecules, it is called 'spontaneous' nucleation which occurs with probability $W_{n}$. When the 'branching' nucleation takes place then a new filament is formed as a branch from the side of an existing filament. The branching nucleation is modeled following the 'dendritic nucleaction' model based on experimental observations [17, 18, 19]. It is known that the activated protein complex Arp2/3 can associate with an existing filament and can nucleate there a new filament as a branch from the mother filament at an angle of about $70^{\circ}$. This leads to the formation of a branched network. In our model on the square lattice, the branching process is implemented as follows. If a G-actin is found to be on the adjacent row to an existing filament, a new daughter filament is created at this site with probability $W_{b r}^{+}$. If there is already a filament in that row, then the nucleation attempt is rejected. Based on the experimental facts [17, 18, 19], the tip of the daughter filament is a plus end, and hence its direction of 


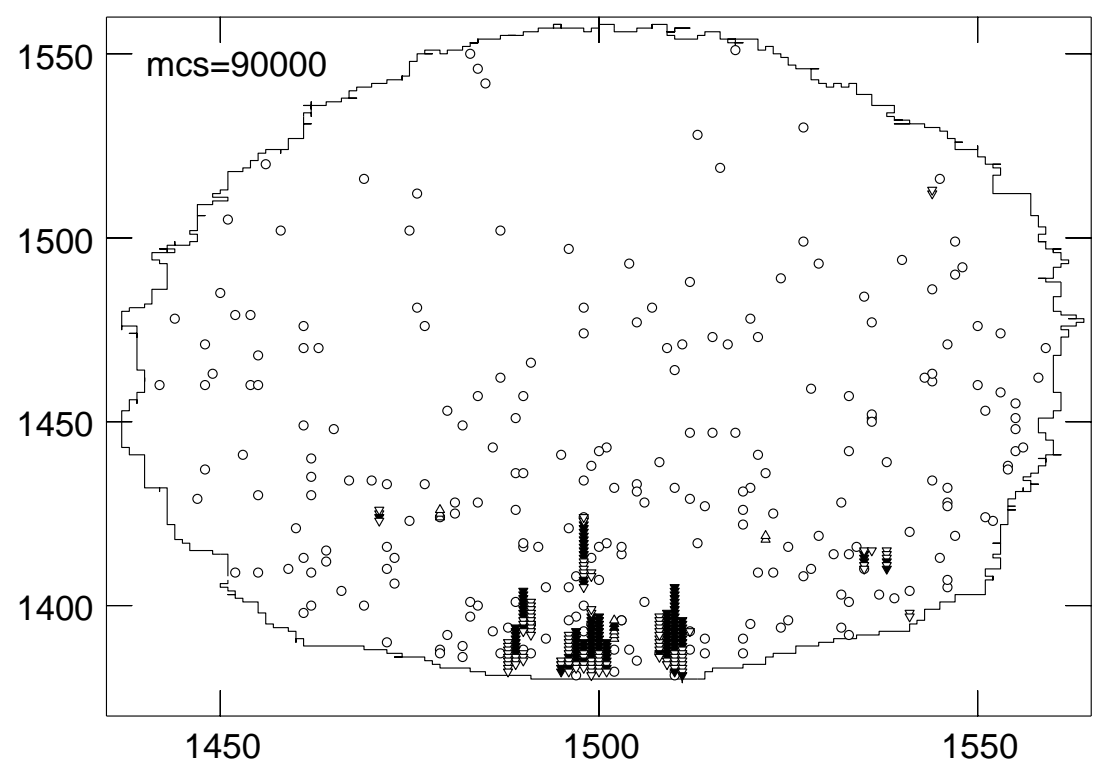

(a)

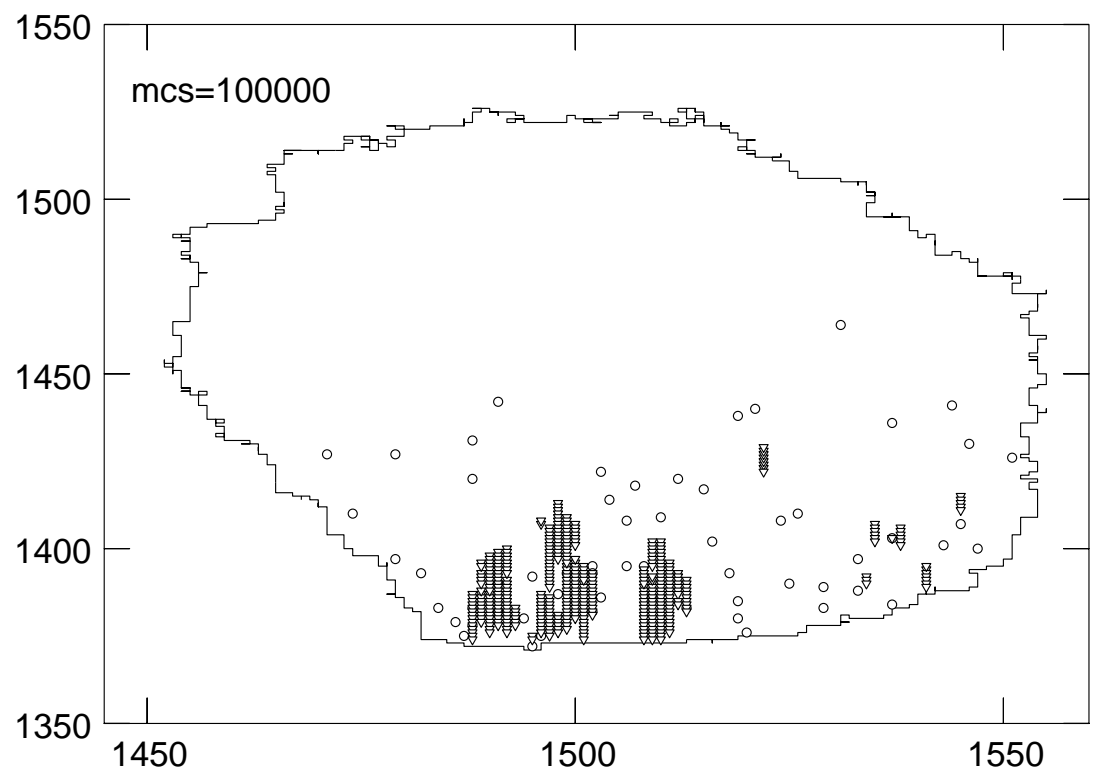

(b)

FIG. 1: Two snapshot ( $a$ and $b$ ) of the model cell. The open circles represent G-actin, the filled triangles represent F-actin molecules. The length of the membrane is $\mathrm{L}=200$, the total number of actin molecules is $\mathrm{N}=500$. 


\begin{tabular}{|l|c|}
\hline Quantity & Value \\
\hline lattice constant $a$ & $5 \mathrm{~nm}$ \\
typical cell size & $100 \times 100$ lattice \\
Monte Carlo step $\tau$ & $0.875 \mu \mathrm{s}$ \\
$W_{n}$ & 0.01 \\
$W_{b r}^{+}$ & 0.1 \\
$W_{b r}^{-}$ & 0.6 \\
$W_{b}^{+}$ & 1 \\
$W_{b}^{-}$ & 0.0012 \\
$W_{p}^{+}$ & 0.11 \\
$W_{p}^{-}$ & 0.02 \\
\hline
\end{tabular}

TABLE I: Model parameters and reaction probabilities.

growth must be the same as the plus end of the mother filament. This implies, that the polarity of a filament, i.e., the vector connecting plus and minus ends, is determined by the spontaneous nucleation process and cannot be changed by dendritic nucleation. In our model, for the sake of simplicity, the spontaneous nucleation creates new filaments only parallel to the Y-axis, pointing at random in one of the two directions.

Reaction Rules. Regarding the reaction rules we follow our earlier work [12]. In brief, the reaction rules are as follows. First we randomly choose one actin molecule. Then for the selected actin molecule, a random choice with a equal probability is made between an association or dissociation process. Then four cases may happen. 1) If for a G-actin molecule the dissiocation process is selected, the step is stopped. 2) If the accociation process is selected for G-actin molecules, then reaction may happen provided the neighbour $\mathrm{G}$ or F-actin exist and the probability $W_{n}$ or $W_{b r}^{+}$or $W_{p}^{+}$or $W_{b}^{+}$fullfill the condition $W>\eta$, where $\eta$ is a random number $0<\eta<1$. 3) If the association process for an F-actin is chosen, then a successful reaction may happen provided a G-actin as a neighbor exists. 4) If a dissociation process of a F-actin molecule is selected, then the process will take place with probability $W_{b}^{-}$or $W_{p}^{-}$or $W_{b r}^{-}$.

Reaction Probabilities and Monte Carlo steps. Since the experimental reaction rates $k_{b, p}^{ \pm}$cannot be used directly in simulations, but instead reaction probabilities $W_{b, p}^{ \pm}$, one must establish a relation between them. In order to calculate these probabilities, we define $W_{b}^{+}=1$ for barbed end polymerization 
and calculate $W_{p}^{+}$for the pointed end using the relation

$$
\frac{W_{p}^{+}}{W_{b}^{+}}=\frac{k_{p}^{+}}{k_{b}^{+}},
$$

The same can be done for the depolymerization rates $W_{b, p}^{-}$, which we choose $W_{p}^{-}=0.02$ and calculate $W_{b}^{-}$by using the corresponding relation to Eq.(2.1). The value of $W_{b}^{-}$has been choosen in order to ensure that the filaments are much shorter than the cell size during the simulations. A basic question is how many Monte Carlo steps, $m$, for Brownian motion membrane and G-actins have to be performed between two sucessive chemical rection attempts. Based on the experimentally known reaction rates and the diffusion coefficient of G-actins, a detailed analysis [12] have shown that $m=5$ is an appropriate value. All model parameters are summarized in Table I.

\section{RESULTS AND DISCUSSIONS}

\section{A. Persistent Random walk}

From experimental observations [2, 3, 20] and from simulation studies [12, 21], it is known that Arp2/3induced dendritic nucleation is essential for the appearance of a persistence random walk (PRW) of certain cells. The PRW is characterized by 'stop-and-go' movements : unidirectional motions with almost constant velocity over distances of the order of several cell diameters are separated by localized short time erratic movements. After each interruption of the ballistic motion, the cell continues to move in a different direction. A typical trajectory of the center of our model cell is shown in Fig 2 as an example from our simulations. The persistency of the cell's random walk can be deduced from the time-dependent mean square displacement of the cell

$$
R^{2}(t)=\left\langle[Y(t)-Y(0)]^{2}\right\rangle
$$

which is shown in Fig 3. The dynamics at short and long times are governed by diffusion, whereas for intermediate times, the cell motion exhibits a unidirectional drift during a certain persistence time. The unidirectional motion has been explained as a consequence of the autocatalytic nature [21, 22] of the dendritic polymerization kinetics. The branching processes induce an explosive growth of filaments in a certain direction which leads to membrane protrusions and concomitant cell movements in the same direction. This motion lasts only for a certain persistence time, then the direction of motion changes. In the following subsection we discuss the reasons for this dynamic instability. It should be noted that insights to the physical basis of the cell's persistency would support, in particular, our understanding 


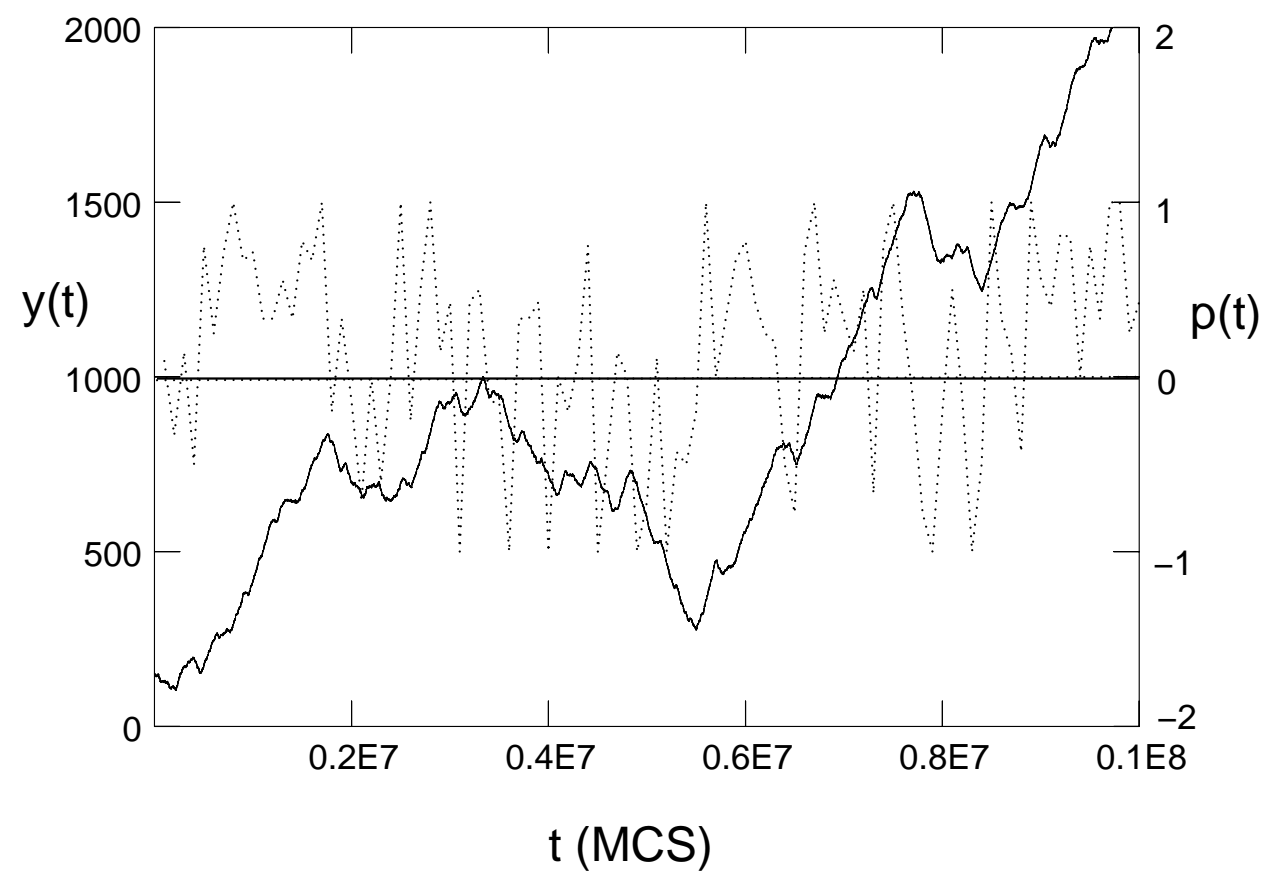

FIG. 2: The trajectory $Y(t)$ (full line, left scale) and polarity $P(t)$ (dotted line, right scale) of a cell exhibiting a persistent random walk, as function of time $t$ in units of Monte Carlo steps, MCS.

of the physical basis of chemotaxis, i.e., the prolongation of the persistency of the cell's motion by an external signal.

\section{B. Dynamic Instability}

The fundamental quantity which governs the migration of the cell is its polarity and its mechanism of self-perpetuation. The cell polarity is macroscopically and dynamically characterized by the formation of leading and trailing membrane edges which are a consequence of the cell's coupling to the polarity of the enclosed actin cytoskeleton. The polarity of the cytoskeleton itself is the average orientation of its filaments, where each actin filament possesses a polarity due to its plus and minus ends. This spontaneous internal polarity of the actin network determines the direction of cell motion and is maintained without external signals (e.g. chemotaxis) only for a certain time (persistent random walk). Specifically, the formation and regulation of cell polarity is achieved by a complex protein signaling network with positive and negative feedback loops. We do not discuss the details here which are beyond the scope of this paper. We note that different signals converge, among others, on the activation of an 


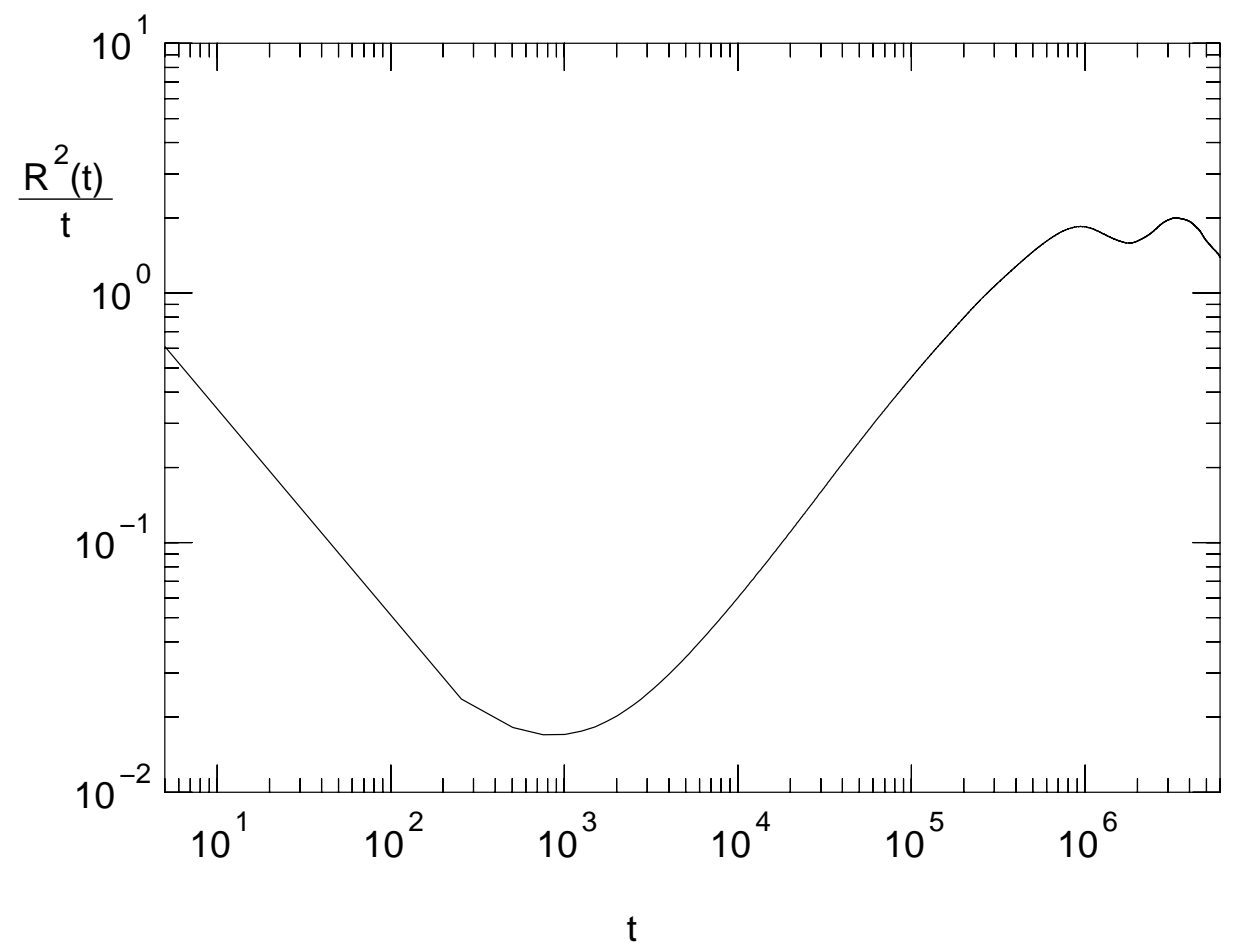

FIG. 3: Typical mean square displacement of a cell exhibiting a persistent random walk.

Arp2/3 protein complex, which leads to branching and the autocatalytic polymerization.

In our model, we define the polarity of the cell by the polarity of the total actin network, i.e., by the difference in the number of filaments pointing in opposite directions,

$$
P=\frac{\left(F_{u p}-F_{d n}\right)}{F_{\text {total }}}
$$

where $F_{u p}$ is the number of filaments pointing in positive $\mathrm{Y}$-direction, $F_{d n}$ in negative $\mathrm{Y}$-direction, and $F_{\text {total }}$ is the total number of filaments. The range of the polarity parameter is $-1 \leq P \leq 1$. The trajectory of this quantity is shown in Fig. 2 (dotted line, right scale). From this result one observes that at certain times, where $P(t)=0$, the cell changes its direction of motion to the opposite as indicated by the corresponding displacements $Y(t)$.

After the analysis of several simulations under different conditions of the model cell, it must be concluded that the cause behind this phenomena is the existence of two antagonistic processes, the spontaneous and the dendritic nucleation processes.

During a spontaneous nucleation process, where two G-actin monomers form a new F-actin filament, the orientation of the new filament is determined at random. This is in contrast to the dendritic nucleation process, where the daughter filament branches off the mother filament and therefore always 
adopts the same growth direction as the mother filament. Hence, spontaneous nucleation changes the sign of polarity, whereas dendritic nucleation increases the absolute value of polarity. The first leads to random motion, whereas the latter prolongs unidirectional motion. Since the polarity fluctuates in time, the spontaneous nucleation may eventually dominate and causes a change of polarity, and hence a change of vectorial motion.

This view is corroborated by simulations of various limiting cases, which are shown in Fig 4.

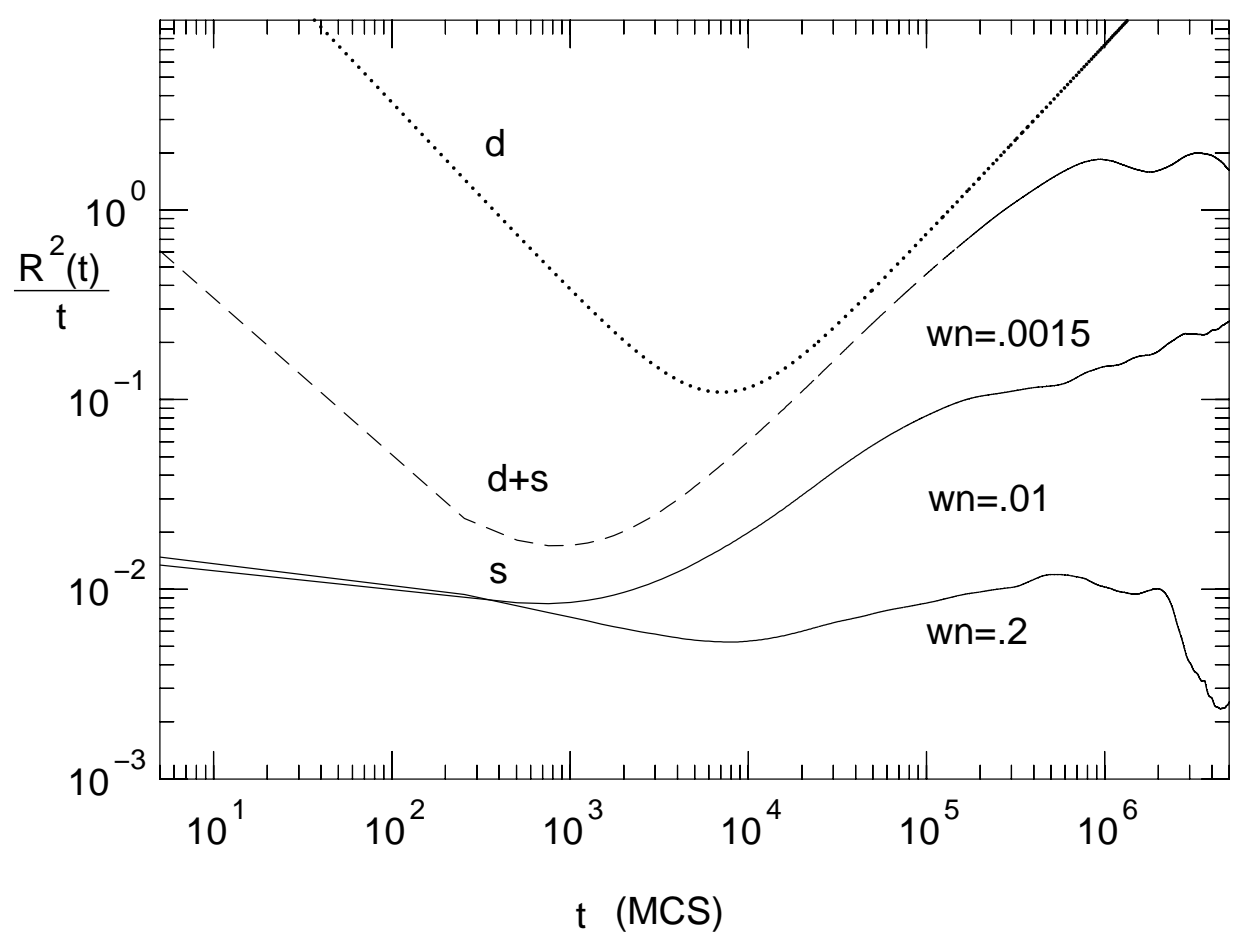

FIG. 4: Mean square displacements of a cell under different conditions and at different probablilities of spontaneous nucleation, wn. 's' represents spontaneous nucleation, 's+d' represents both spontaneous and dendritic nucleation, and 'd' represents dendritic nucleation only.

In one limiting case, the cell motion is caused solely by spontaneous nucleations. The corresponding curve is shown in Fig 4 by the full lines with label 's'. At two different nucleation probabilities, $W_{n}$, the mean square displacements indicate ordinary diffusion, $R^{2}(t) / t \approx$ const., at almost all times. This is expected due to the random orientation of newly established filaments. A very weak persistency may be expected at small nucleation rates.

In the other limiting case, the cell motion is caused solely by dendritic nucleation. Since in this case the orientation of the filaments is preserved, the corresponding curve (dotted line with label 'd') has 
to exhibit a clear drift, $R^{2}(t) / t \sim t$, which takes place for times larger than a characteristic time $\tau_{d} \approx$ $7 \times 10^{3}$. The characteristic time scale $\tau_{d}$ separates periodes of cell advancements and localized cell displacements ('resting'). Since the activation range of Arp2 $/ 3$ extends only a few lattice sites from the membrane, the rapid advancement of the leading edge of the membrane together with the rapid autocatalytic processes of branching near the leading edge deprive this area of available G-actin molecules which are necessary for continuation of these processes. Therefore, actin network and cell remain temporarily, during $t<\tau_{d}$, essentially at rest. While recruiting G-actins by diffusion from the minus to the plus ends of the filaments near the activation zone, the membrane of the cell performes random displacements around the immobile filament network, which leads to time-independent displacements of the cell, $R^{2}(t) / t \sim 1 / t$ at $t<\tau_{d}$. It is still unknown whether this result from simulations is related to the experimentally observed [23] excitation waves of F-actin assembly near the membrane and the correlated cell advancements.

The interesting intermediate case, when a few events of spontaneous nucleation interfere with dendritic nucleations, is shown in Fig⿴囗十 by the broken line with label ' $\mathrm{d}+\mathrm{s}$ '. At a nucleation ratio of $W_{n} / W_{b r}^{+}=0.015$, again a drift of the cell is observed albeit only up to a typical persistence time $\tau_{p} \approx 10^{6}$. At larger times, ordinary diffusion $R^{2}(t) / t=$ const. is observed indicating the dominance of spontaneous nucleation processes. For $t<\tau_{p}$, the mean square displacements are qualitative the same as for the dendritic case 'd'.

\section{SUMMARY AND CONCLUSIONS}

The dynamics of a motile cell with unidirectional and bidirectional branching is studied with treadmilling actin polymerization kinetics. The filaments growing on both sides with treadmilling rates and branching in both directions leads to a persistent random walk whose persistency becomes infinite and leads to unidirectional drift in the case of one side branching. The dynamics are governed by diffusion at short times followed by drift at longer times.

The phenomena is shown to be attributed to two antagonistic nucleation processes during the polymerization of the cell's actin cytoskeleton : the (ordinary) spontaneous nucleation and the dendritic nucleation processes. Whereas spontaneous nucleations generate actin filaments growing in different directions and hence create motions in random directions, dendritic nucleations provides a unidirectional growth. Since dendritic growth exhibits stochastic fluctuations, spontaneous nucleations may eventually compete or even dominate, which results in a reorientation of filament growth and hence a 
new direction of cell motion. The event of reorientation takes place at instants of vanishing polarity of the actin skeleton.

\section{ACKNOWLEDGEMENTS}

B. Nandy would like to thank IBM Research for providing a doctoral fellowship and the Institut für Festkörperforschung at the Forschungzentrum Jülich for hospitality.

[1] D. Weiner, Curr. Opin. Cell Biol. 14, 196 (2002).

[2] D. Bray, Cell Movements, Garland Publ., New York, 2nd. edition, 2001.

[3] D. A. Lauffenburger and A. F. Horwitz, Cell 84, 359 (1996).

[4] T. D. Pollard and G. G. Borisy, Cell 112, 453 (2003).

[5] M.-F. Carlier, C. L. Clainche, S. Wiesner, and D. Pantaloni, BioEssay 25, 336 (2003).

[6] B. Alberts et al., Essential Cell Biology, Garland Publishing, Inc., New York, 1998.

[7] J. Theriot and T. Mitchison, Nature 352, 126 (1991).

[8] J. Lee, A. Ishihara, J. Theriot, and K. Jacobson, Nature 362, 167 (1993).

[9] A. B. Verkhovsky, T. M. Svitkina, and G. G. Borisy, Curr. Biol. 9, 11 (1999).

[10] S. Leibler, R. P. Singh, and M. E. Fisher, Phys. Rev. Lett. 59, 1989 (1989).

[11] R. Sambeth and A. Baumgaertner, J. Biol. Systems 9, 201 (2001).

[12] S. Satyanarayana and A. Baumgaertner, J.Chem.Phys. 121, 4255 (2004).

[13] D. J. Webb, J. T. Parsons, and A. F. Horwitz, Nature Cell Biol. 4, 97 (2002).

[14] K. A. DeMali and K. Burridge, J. Cell Sci. 116, 2389 (2003).

[15] A. Wegner, J. Mol. Biol. 108, 139 (1976).

[16] Y. L. Wang, J. Cell Biol. 101, 597 (1985).

[17] T. M. Svitkina and G. G. Borisy, J. Cell Biol. 145, 1009 (1999).

[18] K. J. Amann and T. D. Pollard, Nature Cell Biol. 3, 306 (2001).

[19] T. D. Pollard and C. C. Beltzner, Curr. Opin. Struct. Biol. 12, 768 (2002).

[20] D. Shreiber, V. Barocas, and R. Tranquillo, Biophys. J. 84, 4102 (2003).

[21] R. Sambeth and A. Baumgaertner, Phys. Rev. Lett. 86, 5196 (2001).

[22] D. Pantaloni, R. Boujemaa, D. Didry, P. Gounon, and M.-F. Carlier, Nature Cell Biology 2, 385 (2000). 
[23] M. G. Vicker, Biophysical Chemistry 84, 87 (2000). 